Crit Pathw Cardiol. 2012 December ; 11(4): 218-219. doi:10.1097/HPC.0b013e318266e687.

\title{
ECG with a Twist
}

\author{
Wayland Lim, MD, Nipavan Chiamvimonvat, MD, and Ezra A. Amsterdam, MD \\ Division of Cardiovascular Medicine, University of California (Davis) Medical Center, Sacramento, \\ CA
}

\begin{abstract}
This report presents a 55 year old man admitted for respiratory failure who was found to have a brief run of polymorphic ventricular tachycardia (PVT) with normal QT interval. The importance of differentiating PVT due to torsades de pointes and PVT with normal QT interval is emphasized. This distinction is crucial because of the differing etiologies and management of these arrhythmias.
\end{abstract}

\section{Presentation}

A 55 year old male was admitted for hypercapnic respiratory failure (obesity hypoventilation syndrome). On the $7^{\text {th }}$ hospital day, telemetry monitor showed recurring 5-7 beat episodes of polymorphic wide QRS tachycardia (WCT) which was also captured on 12lead electrocardiogram (ECG) (figure 1). The rhythm was interpreted as ventricular tachycardia (VT). The patient's history of schizophrenia precluded complete assessment of symptoms but there were no apparent associated symptoms or alteration in objective findings. His medical history included hypertension, schizophrenia, obesity, obstructive sleep apnea, depression, and chronic lung disease. He also had an extensive smoking history. There was no recent reported alcohol or illicit drug use and family history was unobtainable.

\section{Assessment}

Physical examination on the day of the WCT (figure 1) revealed heart rate $60 / \mathrm{min}$, blood pressure $127 / 70 \mathrm{~mm} \mathrm{Hg}$, respirations $20 / \mathrm{min}$ with $93 \%$ oxygen saturation on ambient air. There was no jugular venous distension, cardiac rhythm was regular and no murmurs, gallops, or rubs were heard. Chest examination revealed diffuse wheezes. There was no edema. Medications included two antipsychotic drugs (aripiprazole $5 \mathrm{mg}$ daily and quetiapine $150 \mathrm{mg}$ daily). Laboratory data revealed serum potassium of $3.0 \mathrm{mEq} / \mathrm{l}$ and magnesium of $2.2 \mathrm{mEq} / \mathrm{l}$.

Ten months prior to admission, the patient was hospitalized for dyspnea and altered mental status. At that time, he had a peak troponin I of $0.22 \mathrm{ng} / \mathrm{ml}$ (reference $₫ 0.04 \mathrm{ng} / \mathrm{ml}$ ). Cardiac catheterization showed mild coronary artery disease $(<30 \%$ stenosis of the left circumflex artery) and elevated pulmonary artery pressure $(52 / 26 \mathrm{~mm} \mathrm{Hg})$, which was attributed to his chronic pulmonary disease. Pulmonary capillary wedge pressure was $14 \mathrm{~mm} \mathrm{Hg}$. Left ventriculography demonstrated low normal ejection fraction (55\%).

Correspondence to: Ezra A. Amsterdam, MD, Division of Cardiovascular Medicine, University of California (Davis) Medical Center, 4860 Y Street, Suite 2820, Sacramento, CA 95817, Tel: 916-734-5191, Fax: 916-734-8394, eaamsterdam@ ucdavis.edu.

The authors express their appreciation to Brittany Chatterton, BS for her excellent assistance.

Conflict of Interest and Source of Funding: None to declare. 


\section{Diagnosis}

WCT is defined as $\geq 3$ consecutive QRS complexes in which the QRS duration is $\geq 120$ $\mathrm{msec}$ and the rate is $>100 / \mathrm{min}$. These tachycardias may be of ventricular or supraventricular origin, representing VT or supraventricular tachycardia (SVT) with aberrant conduction, respectively. Although up to $80 \%$ of $\mathrm{WCT}$ are $\mathrm{VT}^{1}$, it is essential to precisely identify the rhythm because the two representations of WCT have widely differing clinical and therapeutic implications. Several algorithms have been developed to aid in distinguishing VT and SVT with aberration but none are completely reliable. A history of structural heart disease strongly favors VT but the ECG is the most important tool in this diagnostic quest. Among the simplest criteria favoring VT is QRS duration >140 msec with right bundle branch block configuration and $>160 \mathrm{msec}$ with left bundle branch block morphology. Atrioventricular dissociation, fusion beats, and capture beats are also important indicators of VT.

In our patient, the contrast between the normal, narrow baseline QRS complexes and the wide, bizarre, beat-to-beat changing QRS complexes is evident (figure 1) and virtually establishes the latter as ventricular in origin. The most striking feature of the patient's WCT is its polymorphic configuration which is consistent with, but not diagnostic of, torsades de pointes (TdP). TdP is defined by 1) QRS complexes that exhibit a characteristic, continuously changing morphology as they appear to twist around an imaginary line and 2) an antecedent long QT interval (LQT) ${ }^{2}$. An increasing list of drugs has been implicated in the acquired form of TdP, including antipsychotic agents, such as our patient was taking ${ }^{3}$. Associated electrolyte abnormalities also promote the arrhythmia. Although our patient had polymorphic VT (PVT) which fulfilled the morphologic criterion for TdP, it was associated with normal QTc (422 msec), thereby precluding the diagnosis of TdP.

PVTs can be classified according to whether they are associated with LQT or normal QT, as in our patient (figure 1). Discrimination between TdP and PVT with normal QT is essential for appropriate understanding and management of these arrhythmias. Figure 2 illustrates the ECG of TdP in a different patient; note the long QT interval preceding onset of PVT.

The most common type of PVT is associated with structural heart disease and a normal QT interval, although it also occurs in patients with structurally normal hearts. Our patient had pulmonary hypertension but no significant coronary artery disease or left ventricular dysfunction. Mechanisms of PVT (normal QT) include myocardial ischemia, electrolyte abnormalities, mutations of the cardiac sodium channel (Brugada syndrome), and the ryanodine receptor (catecholaminergic PVT). Although this arrhythmia may be asymptomatic and detected on ambulatory monitoring, it can degenerate into ventricular fibrillation. In our patient, the electrolyte abnormality (hypokalemia) may have contributed to PVT.

\section{Management}

The patient received potassium repletion to a goal greater than $4.0 \mathrm{mEq} / \mathrm{l}$. Serum magnesium was $\geq 2.2 \mathrm{mg} / \mathrm{dL}$ during the hospitalization. PVT did not recur during the remainder of the patient's hospital course. His antipsychotic medications were continued without dose adjustment, since the arrhythmia was not considered TdP.

This case illustrates the importance of 1) distinguishing PVT with normal QT from TdP and 2) recognizing the differences in management of the two arrhythmias. This approach enhances rational management and will avoid unnecessary and potentially harmful interventions. 


\section{References}

1. Brugada P, Brugada J, Mont L, et al. A new approach to the differential diagnosis of a regular tachycardia with a wide QRS complex. Circulation. 1991; 83:1649-59. [PubMed: 2022022]

2. Kaufman ES. Mechanisms and clinical management of inherited channelopathies: long QT syndrome, Brugada syndrome, catecholaminergic polymorphic ventricular tachycardia, and short QT syndrome. Heart Rhythm. 2009; 6(8 Suppl):51-5. [PubMed: 19121800]

3. Harrigan EP, Miceli JJ, Anziano R, et al. A randomized evaluation of the effects of six antipsychotic agents on QTc, in the absence and presence of metabolic inhibition. J Clin Psychopharmacol. 2004; 24:62-9. [PubMed: 14709949] 


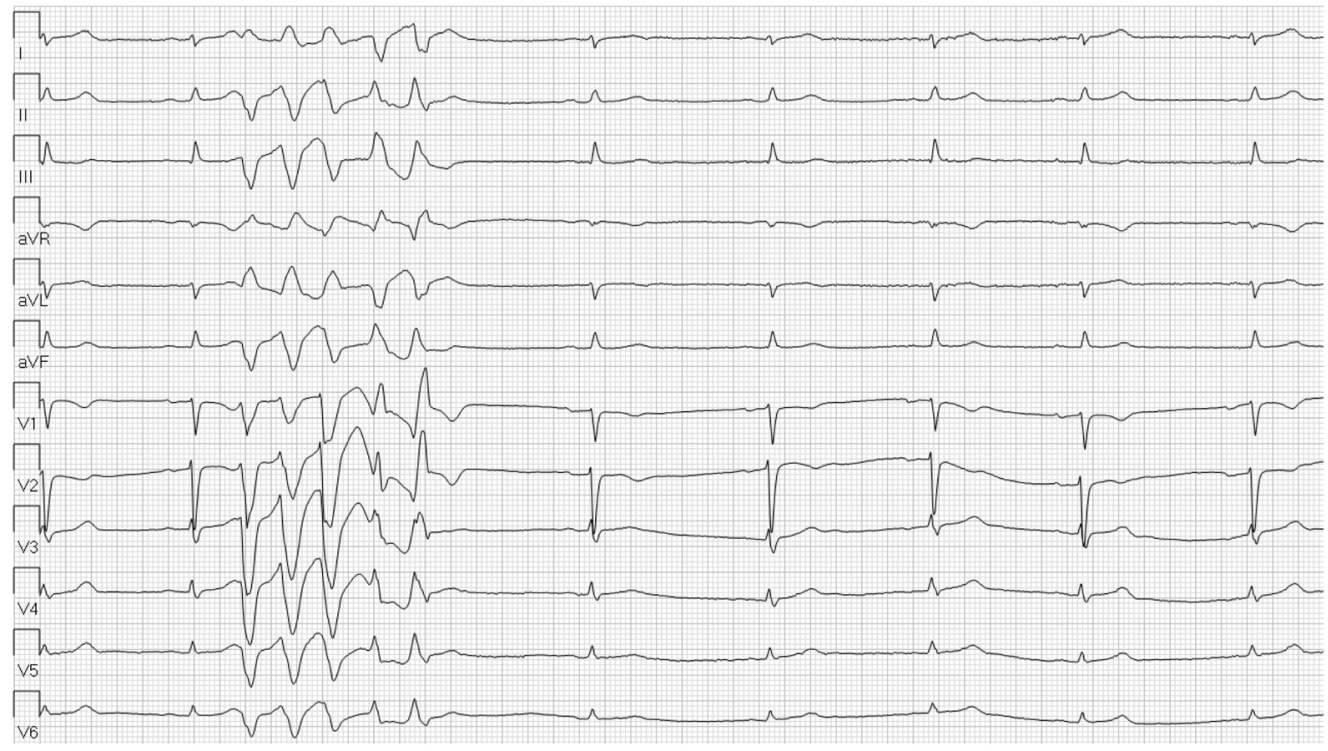

Figure 1.

Electrocardiogram on the day of consulation capturing five beats of polymoprhic ventricular tachycardia. The QTc of the complexes preceding and after the event are 422 and $428 \mathrm{~ms}$, respectively. 


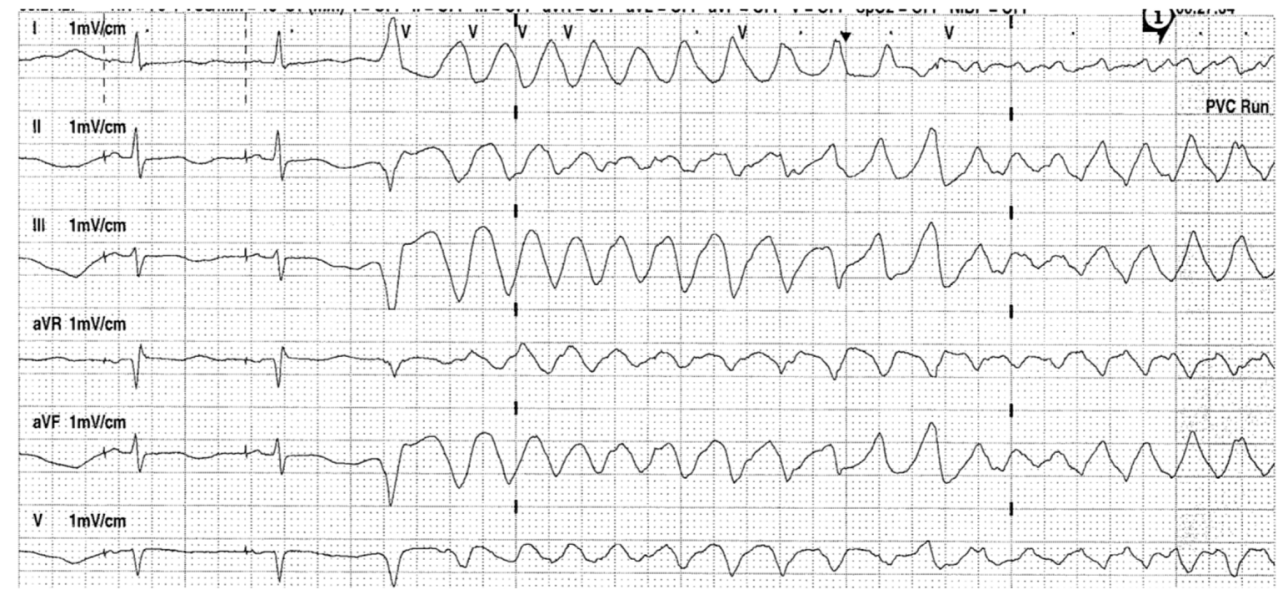

Figure 2.

Telemetry recording of a different patient with two atrial paced complexes with prolonged QTc $(560 \mathrm{~ms})$ followed by torsades de pointes. This was terminated by a shock from the patient's implanted cardioverter- defibrillator. 